\title{
Micro-Arcing and Arc Erosion Minimization Using a DC Hybrid Switching Device
}

\author{
Jonathan Swingler and John W. McBride
}

\begin{abstract}
Hybrid switching devices utilize the advantages of both conventional electrical contacts and solid state electronics to minimize arcing during opening and closing operations. This can result in higher reliability and reduces the need for high cost specialist contact materials. The hybrid switch does not eliminate arcing completely, due to the inductive nature of circuits; micro-arcing is known to occur.

An experimental dc hybrid switching device is introduced which minimizes arcing for $42 \mathrm{~V}$ applications. The characteristics of micro-arcing are investigated to determine the factors which influence the duration of micro-arcs. Surface profiling techniques are used to determine low level contact erosion. The magnitude of contact erosion is related to the micro-arcing.
\end{abstract}

Index Terms-Arc erosion, hybrid switch, micro-arcing.

\section{INTRODUCTION}

$\mathbf{T}$ HE method of actuation of switching contacts has a direct influence on contact performance and reliability, [1]. Welding of the contacts can occur [2] and has been shown to occur at the beginning of separating the contacts under an arc [3]. The arc also causes contact erosion and transfer of material [4] leading to degradation of the system. All this has led to the development of specialist alloys [1], [5]. In medium/high power devices, arc control mechanisms are used; for example, de-ionising chambers, these increase the size and weight of the device. This is particularly undesirable in aerospace applications. Semiconductor devices have some advantages over conventional switching methods [6], as they provide both arc-less and faster switching. However there are some limitations in using semiconductors, where the conventional mechanical device offers improved performance. The conventional mechanical device has a higher degree of isolation in the open (off) state requiring high breakdown voltages to conduct, and a lower volt-drop across the device when in the closed (on) state.

A hybrid switching discussed here combines the advantages of both technologies [7]. The main advantages of the hybrid switch are: 1) arc minimization to improve performance, reliability and reduce the need for expensive specialist contact materials, 2) faster switch response, and 3) physical electrical isolation. Semiconductors have been the favoured technology for hybrid switching but there are alternatives technologies which have been investigated, [8]-[14].

Manuscript received November 17, 2004. This work was recommended for publication by Associate Editor M. Braunovic upon evaluation of the reviewers comments.

The authors are with the School of Engineering Sciences, University of Southampton, Southampton S017 1BJ, U.K. (e-mail: swingler@ soton.ac.uk).

Color versions of one or more of the figures in this paper are available online at http://ieeexplore.ieee.org.

Digital Object Identifier 10.1109/TCAPT.2008.921640

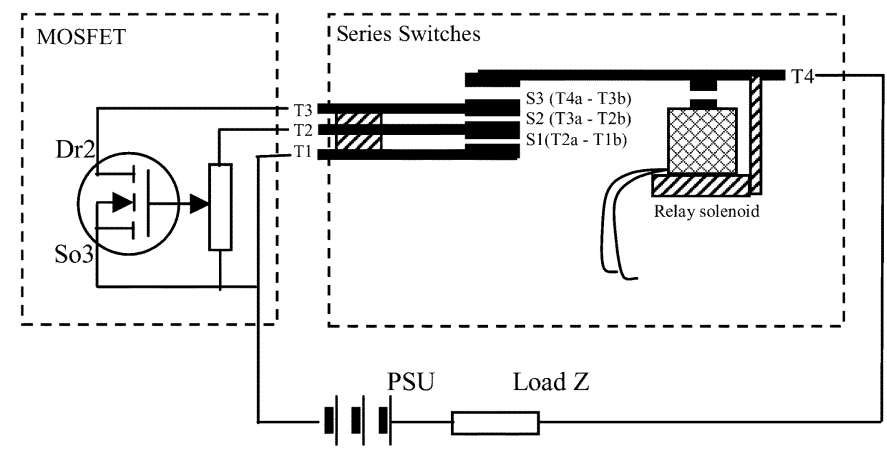

Fig. 1. Hybrid switch connected to a power supply and load.

Micro-arcing has been shown to occur in hybrid switching devices, which leads to erosion at the contact surface [8]. The phenomenon occurs on opening, due to commutation of the current from the separating contacts to the semiconductor circuit. This study investigates the impact of micro-arcing on the erosion characteristics of hybrid switch contacts.

A hybrid switch is presented for low voltage automotive applications, since increasing demands on the power systems in automotive systems has led to the application of system voltages above the 12-14 V standard.

\section{EXPERIMENTAL ARRANGEMENT}

\section{A. Hybrid Switch}

The hybrid switching device presented consists of two groups of components as illustrated in Fig. 1: The mechanical components of contacts arranged as a series of switches and the electronic components utilising a MOSFET arrangement fitted in parallel to switches S1 and S2.

1) Series Switches: A series of contacts makes up three switches which are operated by a solenoid such that they open in sequence: S1, S2 and S3. They close in the reverse sequence: $\mathrm{S} 3, \mathrm{~S} 2$ and $\mathrm{S} 1$. Switch $\mathrm{S} 1$ is connected to terminal $\mathrm{T} 1$ and $\mathrm{T} 2$ as in Fig. 1 (Contacts T1b and T2a), S2 is connected to terminals $\mathrm{T} 2$ and $\mathrm{T} 3$ (Contacts $\mathrm{T} 2 \mathrm{~b}$ and $\mathrm{T} 3 \mathrm{a}$ ), and $\mathrm{S} 3$ is connected to terminals T3 \& T4 (Contacts T3b and T4a). Switches S1 and $\mathrm{S} 2$ turns the MOSFET on/off on opening/closing. Switch S3 is an isolation switch [7].

2) MOSFET Arrangement: A MOSFET and potential divider are connected to the three switches as illustrated in Fig. 1. This is used to bypass the current away from the contacts and switch the current during opening and closing (break and make).

Fig. 1 shows these two groups of components, along with a nominal $42 \mathrm{~V}$ direct-current (dc) power supply and a load, $Z$. 
The power supply consists of three $12-14 \mathrm{~V}$ batteries giving an open circuit voltage of $38-42 \mathrm{~V}$.

\section{B. Experimental Procedure}

The hybrid switch is tested under three different states to evaluate the extent of contact erosion in each of these states: 1) opening and closing the switch contacts under load, 2) opening the contacts under load and closing with the power supply disabled, and 3) closing the contacts under load conditions and opening with the power supply disabled. New contacts are used for each test.

Up to 2000 open and close operations are conducted upon the hybrid device for the particular load conditions. Pure silver contact materials are used.

Resistive Load: The load is set to give 8 A using a $4.75 \Omega$ resister bank. The contacts are open/closed at a typical velocity of $1 \mathrm{~ms}^{-1}$.

Inductive Load: Under inductive load a $38 \mathrm{mH}$ inductor is placed in series with the resistance. The contacts are open/closed at a velocity significantly reduced to enable the MOSFET to turn off $\left(0.1 \mathrm{~ms}^{-1}\right)$.

\section{Analysis Procedure}

1) Voltage/Current Characteristics: The voltage drop is measured across the whole hybrid switch (between T1 and T4) and across the MOSFET (between T1 and T3). The current through the hybrid switch is monitor using a current clamp probe at terminal T4.

2) Contact Surface Analysis: A non-contact 3-D profiler is used to analyses the surface condition of the contacts [15]. This is used to determine the extent of erosion in terms of volumetric deposition and removal. Volume measurements are used instead of mass measurement due to the small changes caused by micro arcing, and the fact that material is moved around the surface of a contact and not necessary lost from the surface [16]. The minimum volume measurable in the arrangement and software used is $10^{-15} \mathrm{~m}^{3}$.

\section{RESULTS}

\section{A. Voltage and Current Characteristics}

1) Resistive Load Opening Characteristics: Fig. 2 shows three typical plots of voltage and current characteristics of the hybrid switch under resistive load on opening. Three events are identified, indicating when the three switches S1, S2, and S3 open.

Fig. 2(a) is a plot of the voltage drop across the whole hybrid switch (measured between T1 and T4). Two of the three events are identified on this profile. At $\sim 2 \mathrm{~ms}$, switch $\mathrm{S} 1$ starts to open probably causing a molten bridge to form between the contact surfaces (T1b and T2a). This is indicated in the data [not shown on Fig. 2(a)] with a slight increase in voltage to $1.0 \pm 0.2 \mathrm{~V}$ before the jump to $12.0 \pm 2.0 \mathrm{~V}$ as shown on the graph. This jump to $12 \mathrm{~V}$ lasts for between $0.8-4.0 \mu$ s probably indicating an arcing event and then reduces to $4.0 \pm 0.1 \mathrm{~V}$. This arcing event is shown in detail in Fig. 3. The $4 \mathrm{~V}$ drop indicates that the MOSFET is turned on.

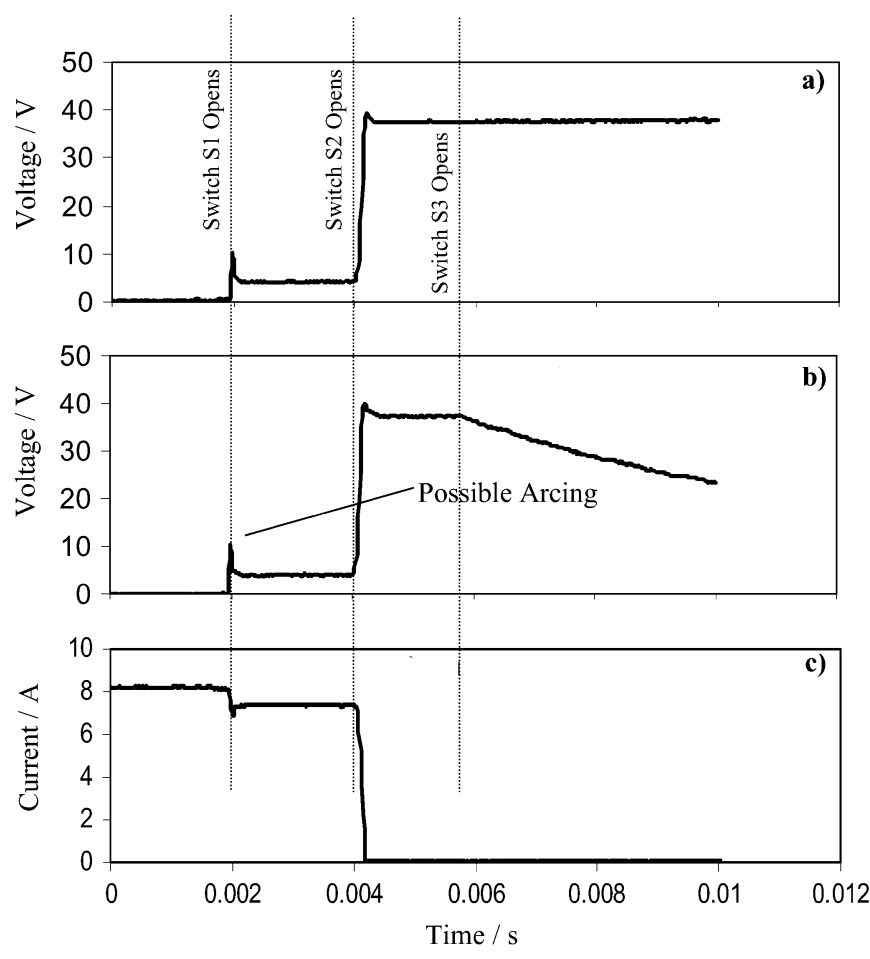

Fig. 2. VI characteristics on opening a circuit of a resistive load.

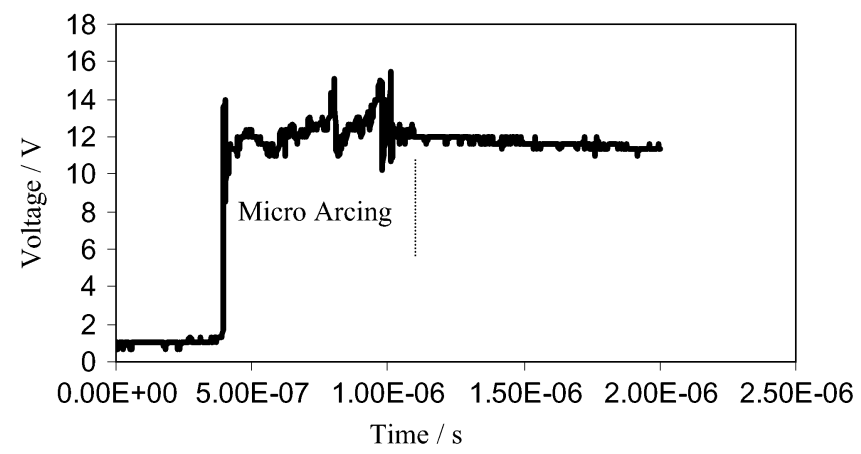

Fig. 3. Opening switch S1.

The next event occurs at $\sim 4 \mathrm{~ms}$ when switch $\mathrm{S} 2$ starts to open. This turns off the MOSFET which takes $0.2 \mathrm{~ms}$ for the current to reach 0 A in Fig. 2(c). The voltage drop across the hybrid switch then increases to the open circuit voltage and remains at that value $(38 \mathrm{~V})$.

Fig. 2(b) is a voltage profile between terminals T1 and T3, it shows a similar profile to Fig. 2(a). The third event, clearly seen on Fig. 2(b), is the opening of switch S3. Switch S3 is an isolation switch, isolating T3 (and the MOSFET Drain terminal) from the load. After the third event, the voltage developed across the MOSFET decays to zero.

Fig. 2(c) shows the load current. The current is zero when the MOSFET is switched off at $\sim 4 \mathrm{~ms}$ by Switch S2 opening.

2) Resistive Load Closing Characteristics: Fig. 4 shows three typical plots of the voltage and current characteristics of the hybrid switch under resistive load, on closing. Three events of switch closure can be seen along with other events due to contact bounce. 


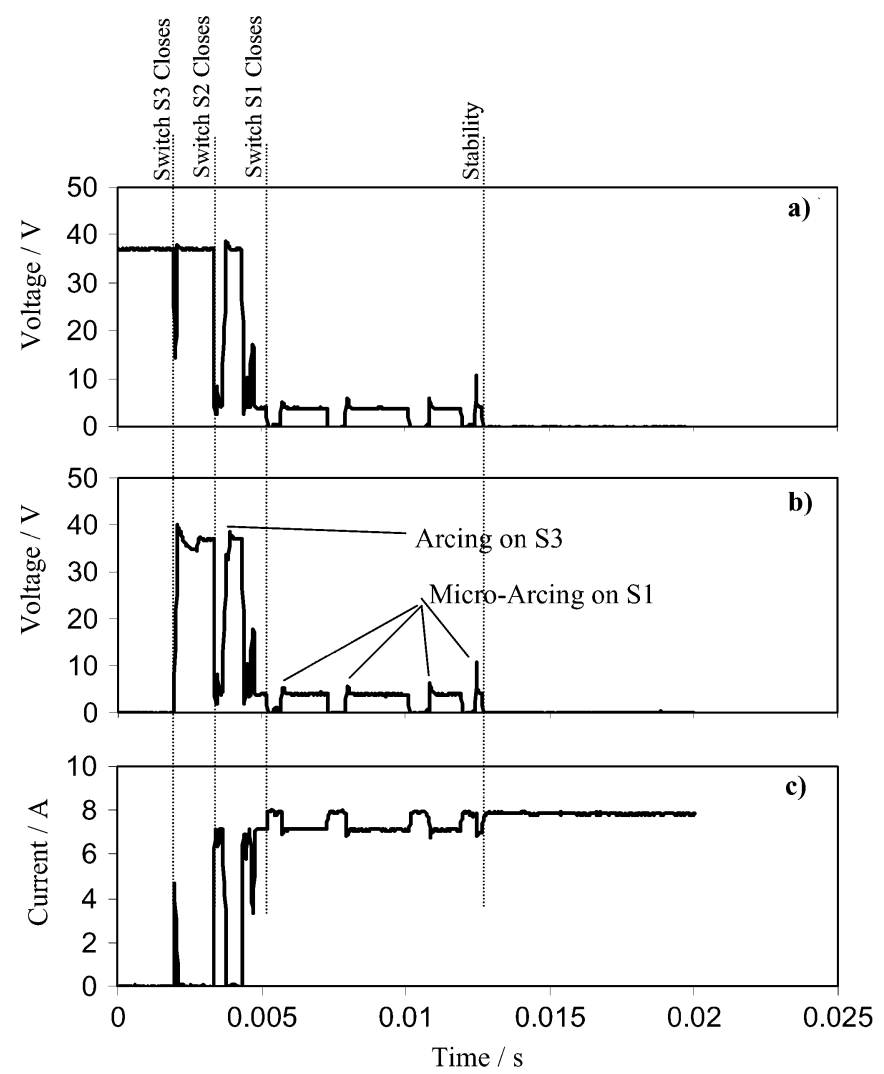

Fig. 4. VI characteristics on closing a circuit of a resistive load.

Fig. 4(a) shows that as switch S3 closes there is a reduction in voltage from the open circuit voltage $38 \mathrm{~V}$ to $\sim 15 \mathrm{~V}$. At this point the voltage across the MOSFET (Fig. 4(b), the voltage between $\mathrm{T} 1$ and $\mathrm{T} 3$ ) increases to the open circuit value. Fig. 4(c) shows an increase of current for $1.0 \pm 0.1 \mathrm{~ms}$ causing the MOSFET to polarize.

Fig. 4(b) indicates an event after the switch S3 closes and before $\mathrm{S} 2$ closes. This is the bounce event of switch S3. Fig. 4(b) indicates a reduction of voltage from open circuit to $35 \mathrm{~V}$ and back to a plateau of $38 \mathrm{~V}$.

The closure of switch S2 is seen on all three plots. During this event the MOSFET switches on permitting a current (7 A) to flow through the MOSFET. Two bounce events of switch S2 occur after this resulting in the current being switch off and on. Switch S1 closes to permit the current to flow through the series switches bypassing the MOSFET, allowing $8 \mathrm{~A}$ to flow. This change in current level from 7 to $8 \mathrm{~A}$ is clearly depicted in Fig. 4(c). Four bounce events of switch S1 are indicated on the plots in Fig. 4 with a voltage plateau of $\sim 4 \mathrm{~V}$, below the minimum arcing voltage.

Fig. 4(a) and (b) indicate possible arcing events at the beginning of each bounce by a voltage peak. These events last for $50 \pm 5 \mu \mathrm{s}$.

3) Inductive Load Opening Characteristics: Fig. 5 is a typical plot of the voltage drop across T1 and T3 and the current through the circuit of an inductive load. Three features are markedly different from the data presented in Fig. 3 of the resistive load condition. First, the voltage drop has exceeded the power supply voltage to a value of $\sim 75 \mathrm{~V}$. Secondly, when the MOSFET turns

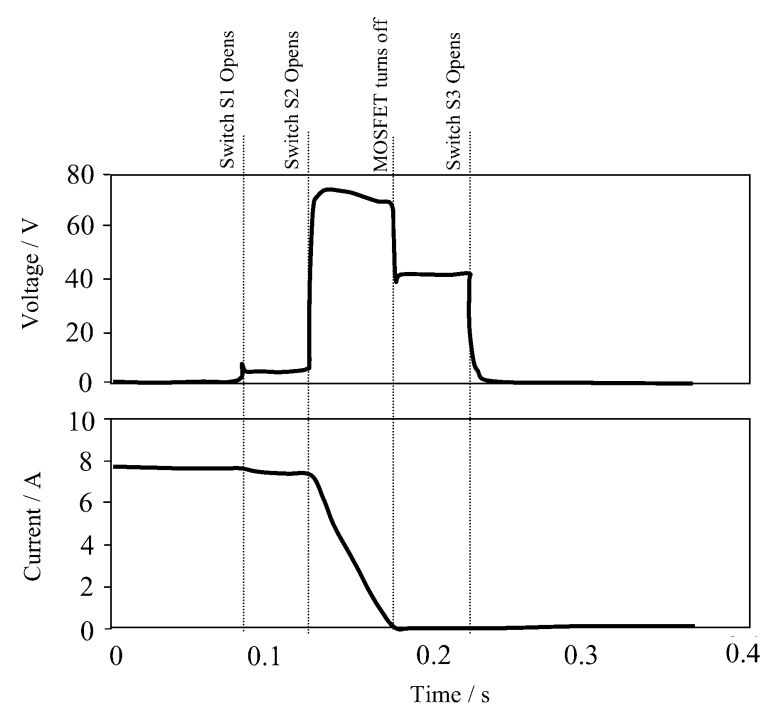

Fig. 5. Characteristics with an inductive load.

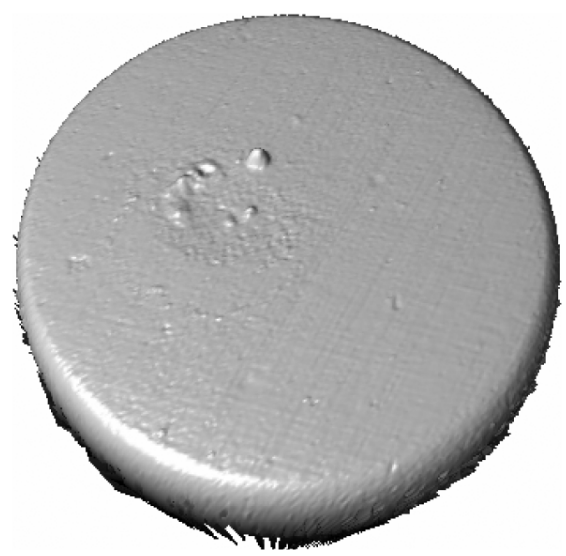

Fig. 6. 3-D profile of eroded contact with conventional switch.

off, this is indicted in the voltage profile with the high voltage level coming to the open circuit voltage. The third feature to note is the long switching time of the MOSFET of $100 \pm 5 \mathrm{~ms}$. It should be noted that if switch S3 is opened before the MOSFET is turned off, excessive arcing occurs between $\mathrm{T} 3$ and $\mathrm{T} 4$.

\section{B. Erosion Characteristics}

It should be noted that the hybrid design tested has not been optimized to reduce contact bounce.

1) Erosion of a Conventional Switch: Fig. 6 is a 3-D surface profile of a flat silver contact (anode) from a conventional switch which has undergone arcing. The switch used is the hybrid switch with the MOSFET circuit disabled. The contact has undergone five opening and closing operations with a second contact (not shown) at $8 \mathrm{~A}$ resistive loading at $38 \mathrm{~V}$ make and break. Arcing took place during this test resulting in erosion on the contact as indicated on Fig. 6. Material is both removed and deposited in the erosion region toward the middle of the contact.

A micrograph of the erosion region is given in Fig. 7. There is extensive discoloration around and in the eroded area. The amount of material removed from the surface of this contact is found to be $2.8 \times 10^{-3} \mathrm{~mm}^{3}$ and the amount of material deposited is $2.7 \times 10^{-3} \mathrm{~mm}^{3}$. This gives a removal (and deposit) 


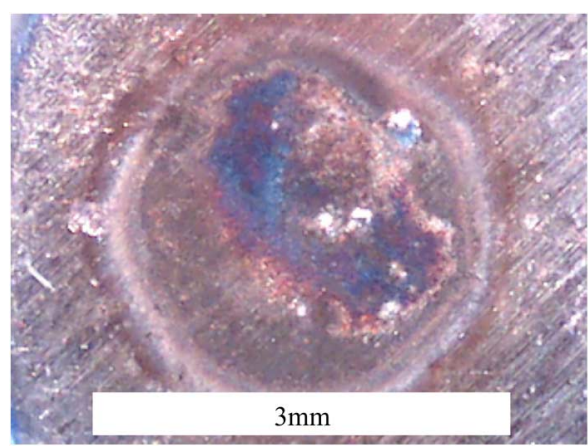

Fig. 7. Arc erosion with conventional switch.
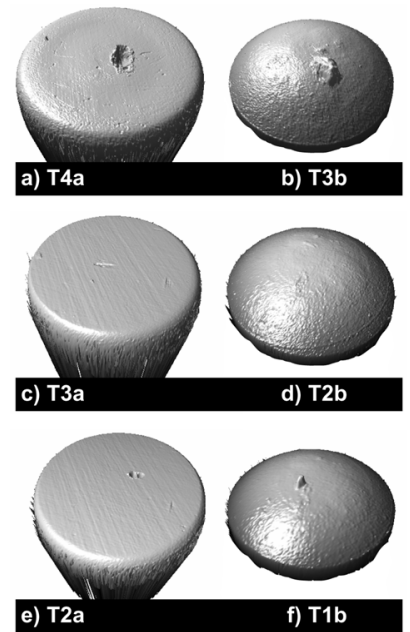

Fig. 8. Hybrid switch contacts after 2000 op at 8 A.

rate of $5.6 \times 10^{-4} \mathrm{~mm}^{3}$ per operation. This is found using the non-contact 3-D profiler as mass measurement would not differentiate between removal and deposition. This result indicates that this is close to a net zero mass change.

2) Erosion on Opening and Closing the Hybrid Switch: Fig. 8 shows six 3-D profiles of the contacts used in the hybrid switch using 8 A resistive loading at $38 \mathrm{~V}$. This test was run for 2000 opening and closing operations under load. Many significant erosion features can be seen on the surfaces with material removal and deposition.

Contact terminals T4a, T3a and T2a are anodes and indicated mainly material removal with some material deposition. Contact terminals $\mathrm{T} 3 \mathrm{~b}, \mathrm{~T} 2 \mathrm{~d}$ and $\mathrm{T} 1 \mathrm{~b}$ are cathodes and indicated erosion with some material removal but more deposition.

Fig. 9 is a micrograph of contact T4a the contact exhibiting one of the greatest amount of erosion of the six contacts. This shows minimal discoloration compared to the convectional switch contact in Fig. 7 which underwent extensive arcing. There is some discoloration in Fig. 9 around the periphery of the eroded area.

The erosion of each contact in the hybrid switch under load conditions at opening and closing is determined using the 3-D profiles [15]. Fig. 10 is a plot of the material removed and deposited on the respective contacts for three different experiments, 1000, 1500 and 2000 open/close operations under resistive load. Each contact showes evidence of deposits and material

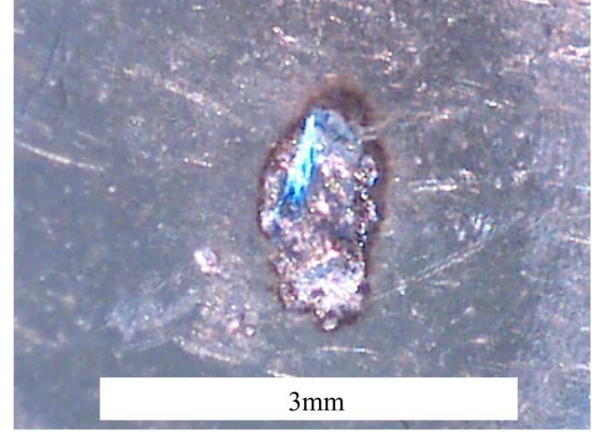

Fig. 9. Erosion of contact terminal T4a.

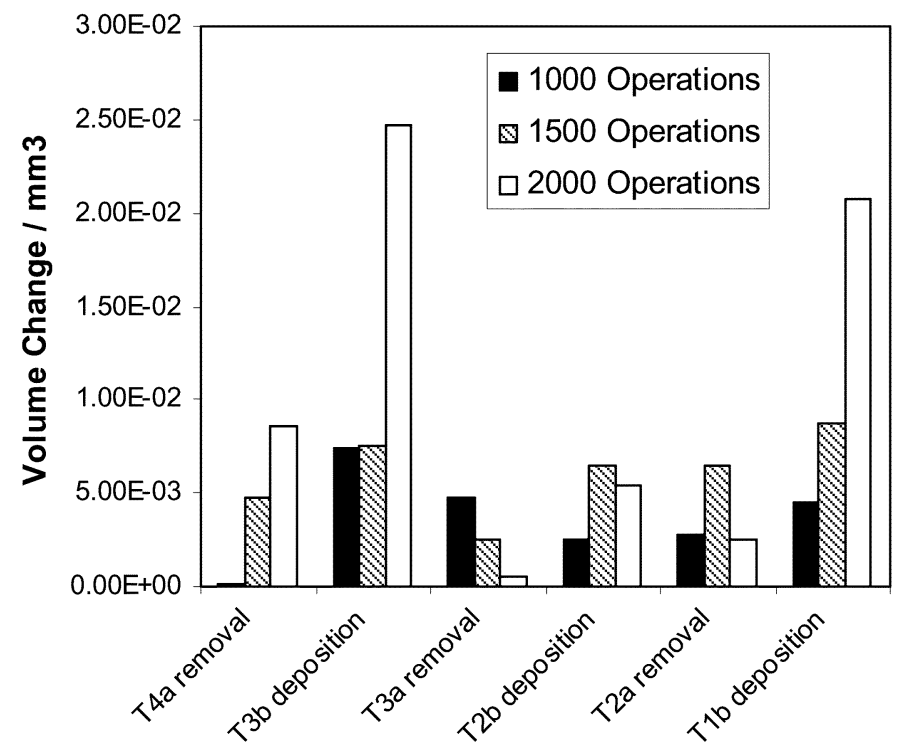

Fig. 10. Contact material removal/deposition.

removal, however the plot is only of the dominate process for a particular contact, material deposition or removal. The worst case contact (T3b) shows a deposition rate of $12.5 \times 10^{-6} \mathrm{~mm}^{3}$ per operation which is 45 times smaller than the conventional switch.

3) Opening Contacts Under Power and Closing Contacts Under Power: Fig. 11 is a bar chart of the material removed and deposited on the respective contacts for 2000 operations for three different experiments, 1) power on when opening and closing contacts, 2) power on when closing contacts but off when opening, and 3) power on when opening but off when closing the contacts. The main feature to observe is the minimal amount of removal and deposition when the contacts are opened under power. The data shown in Fig. 11 does not combine such that the opening contacts under power plus closing contact under power equals opening and closing under power. This is under further investigation.

\section{DISCUSSION}

The hybrid switch operating under resistive loads does not show any evidence of arcing for more than $300 \mu \mathrm{s}$. Only microarcing is exhibited. This is also true for the inductive load when that the MOSFET is given time to switch off by opening the series switches at a slower rate. The contact 3-D profiles show 


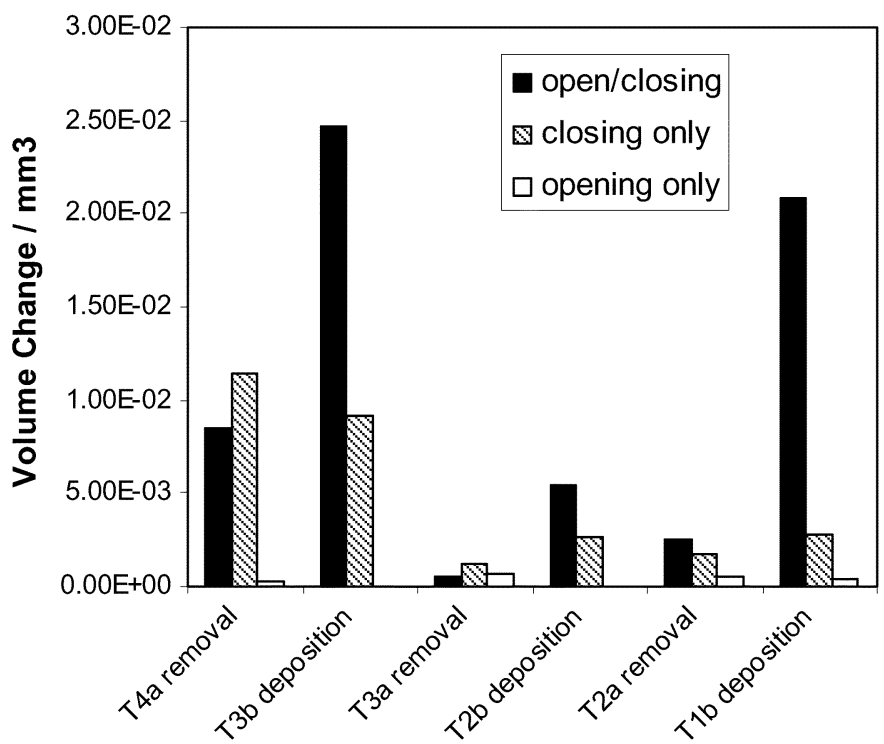

Fig. 11. Contact material removal/deposition for powering on a) opening/ closing, b) closing only, and c) opening only.

evidence of arc erosion with pips and crater formations. Switch S3 has the largest amount of erosion of its contacts followed by switch S1 contacts. There is minimal damage on switch S2 contacts.

Micro-arcing is indicated on the voltage characteristic in Figs. 2 and 4 of the resistive experiments. During the opening operation, micro-arcing is found on switch S1 only with duration of between 1.5-4.0 $\mu \mathrm{s}$. During the closing operation, arcs of up to $50 \mu$ s are observed on switch S1 when the contacts undergo bouncing events. A longer duration of arcing is also observed on switch S3 on closing.

\section{A. Switch S3 Arc Erosion on Closing}

There is no evidence of micro-arcing on opening switch S3 in Fig. 2(a) and (b). Also, Fig. 11 illustrates that the erosion and deposition of opening switch S3 under power is minimal compared to other switches. However on closing switch S3 there is evidence of arcing in Fig. 2(a) and (b). The duration is $200 \pm 10 \mu \mathrm{s}$, see Fig. 12(c) and (d). Fig. 12 is a plot of four graphs showing voltage with time. Fig. 12(a) is a close-up of Fig. 4(b), the voltage drop across terminals T1 and T3. A second experiment is shown in Fig. 12(b) of the voltage drop across terminal T1 and T3. Figs. 12(c) and 10(d) are plot of the voltage drop across the switch S3 acquired by subtracting the data of the voltage drop across T1-T3 from the voltage drop across T1-T4.

The arc appears to develop on switch S3 on closure as the contacts bounce. The two experimental runs shown illustrate that this bounce event can vary in time between $3.3 \mathrm{~ms}$ and $3.8 \mathrm{~ms}$, as in Fig. 12, even though the closure times of switches S3 and $\mathrm{S} 2$ are reproducible.

These bounce events need to be eliminated to prevent arcing and thus improve performance of the hybrid switch. The design of hybrid used in these preliminarily tests has not been optimized. To reduce the bounce between the closing contacts the velocity profile of the actuation can be modified, along with mechanical design linking switches S1, S2, and S3.
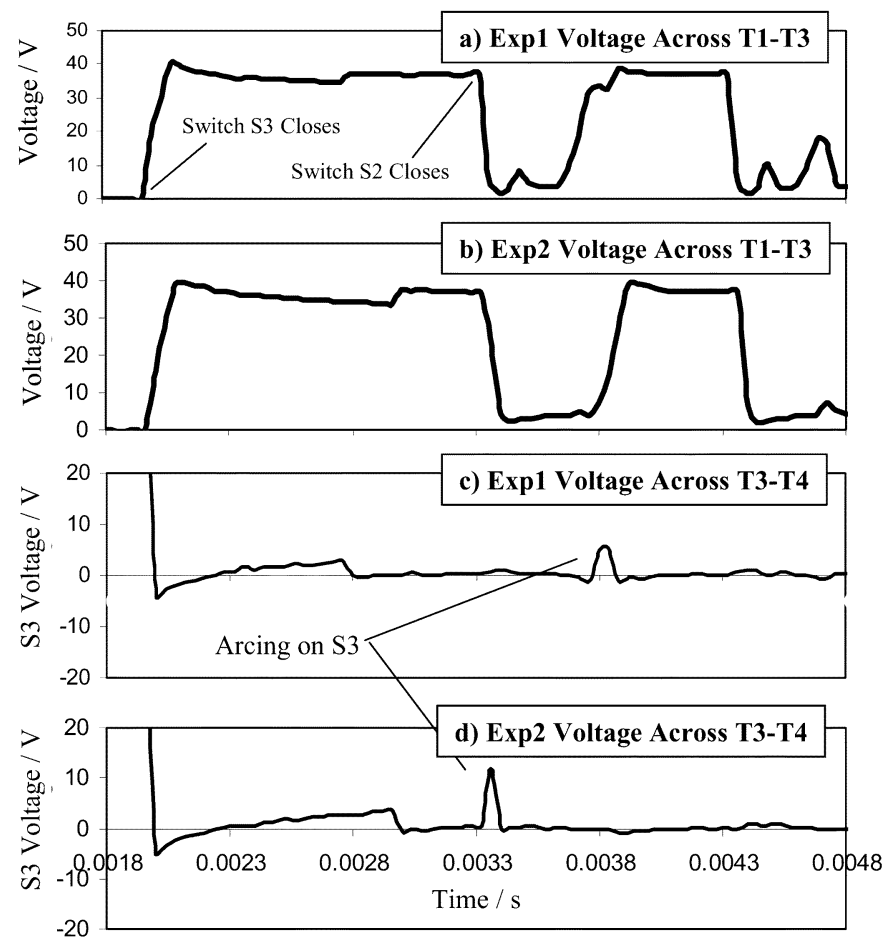

Fig. 12. Arcing on switch S3.

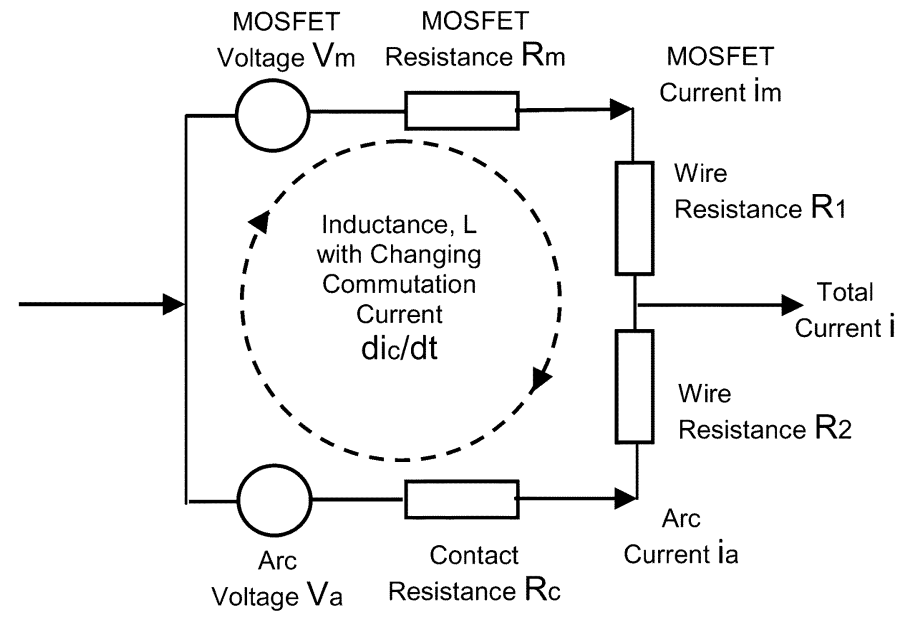

Fig. 13. Equivalent circuit of the commutation circuit.

\section{B. Switch S1 Arc Erosion on Opening and Closing}

There is evidence of micro-arcing on opening and closing switch S1. Fig. 2(b) shows that at the first event there is arcing under the opening operation. Fig. 4(b) shows that there is arcing after each of the four bounce events of switch S1 closing.

On opening the contacts of the hybrid switch, the current travelling through the contacts has to be reduced and directed to the MOSFET part of the circuit. In effect the current through the contacts (and forming the micro-arc) decreases and the current through the MOSFET increases. Fig. 13 is an equivalent circuit of the commutation circuit of the hybrid switch modelling the behaviour of the circuit [8]. This change in current during opening is illustrated in Fig. 13 by $d i_{c} / d t$. In this paper, the model is used to show that miniaturization of the hybrid device will lead to further reduction in micro-arcing. 
A possible method to reduce micro-arcing is to modify this circuit to give a smaller inductance. Reducing the wire lengths between the series switches and the MOSFET arrangement reduces the resistances $R_{1}$ and $R_{2}$, and reduces the inductance $(L)$ of the equivalent circuit. Therefore the rate of change of the commutation current is affected and is expected to result in a shorter duration of micro-arcing which in turn should lead to less erosion. Equation (1) gives the theoretical relationship

$$
\frac{d i_{c}}{d t}=\frac{\left(v_{a}-v_{m}\right)}{L} \text {. }
$$

Since the voltages $v_{a}$ and $v_{m}$ are nearly constant [8], the arcing time can be expressed as in (2), assuming there is no delay time for switching on the MOSFET

$$
t_{a}=\frac{i L}{\left(v_{a}-v_{m}\right)} .
$$

The inductance $L$ can be calculated as a first approximation from (3), for an inductance of a one-turn coil of wire of thickness $s$

$$
L=\mu_{0} \frac{l^{2}}{4 \pi s} .
$$

Combining (2) and (3) gives (4) where $h$ is a constant

$$
t_{a}=\frac{i \mu_{0}}{4 \pi s\left(v_{a}-v_{m}\right)} l^{2}=h l^{2} .
$$

This term shows that the micro-arc duration is a function of the square of the wire length. Halving the wire length is expected to reduce the micro-arc duration to a quarter. It is expected that the hybrid design presented will be miniaturized to reduce the conduction path and also to reduce the bounce phenomena.

\section{CONCLUSION}

An experimental prototype dc hybrid switch presented exhibits only micro-arcing under resistive loads. 3-D surface analysis shows that the contact erosion rate is 45 times smaller than that of a conventional switch. The hybrid switch behaves in a similar manner under inductive loads with only micro-arcing. However, the series switches have to be open and closed at a slower rate to enable the MOSFET to switch the load current.

The hybrid switch consists of two groups of components a series of mechanical switches and a MOSFET arrangement. The switch is considered to be a parallel device because under switching conditions the current is bypassed through a parallel MOSFET circuit away from series switches $\mathrm{S} 1$ and $\mathrm{S} 2$. It should be noted that the isolation switch S3 is in series with the MOSFET arrangement.

The MOSFET is used to bypass the current for a short amount of time during opening or closing, 2-3 ms. Therefore ideally an appropriately low rated MOSFET can be selected for the application.

Improvements in the hybrid switch are proposed to reduce the erosion rate further: a) the reduction in the length of wire leads connecting the components in the switch and b) the minimising of contact bounce.

During the opening operation minimal micro-arcing occurs resulting from the commutation of the load current from the series switches to the MOSFET. Further investigations are underway to reduce this micro-arcing by minimising wiring in the hybrid switch. Consideration will also be given to contact material selection.
Contact bounce is the major course of arcing during closure of the hybrid switch. The series switches are being developed further to reduce bounce.

\section{REFERENCES}

[1] J. Swingler and J. W. McBride, "The erosion and arc characteristics of $\mathrm{Ag} / \mathrm{CdO}$ and $\mathrm{Ag} / \mathrm{SnO} 2$ contact materials under dc break conditions.," IEEE Tran. Compon., Packag., Manufact. Technol. A, vol. 19, no. 1, pp. 404-415, Mar. 1996.

[2] P. Slade, Ed., Electrical Contacts: Principles and Applications. New York: Marcel Dekker, 1999.

[3] N. B. Jemaa, L. Morin, S. Benhenda, and L. Nedelec, "Anodic to cathodic arc transition according to break arc lengthening," IEEE Trans. Compon., Packag., Manufact. Technol. A, vol. 21, no. 04, pp. 559-566, Dec. 1998.

[4] L. Morin, N. B. Jemaa, and D. Jeannot, "Make ARC erosion and welding in the automotive area," IEEE Trans. Compon. Packag. Technol., vol. 23, no. 2, pp. 240-245, Jun. 2000.

[5] N. B. Jemaa, L. Doublet, L. Morin, and D. Jeannot, "Break arc study for the new electrical level of $42 \mathrm{~V}$ in automotive applications?," in Proc. 47th Holm Conf., Montreal, QC, Canada, Sep. 2001, pp. 50-55.

[6] D. R. Grafham and J. C. Hey, Eds., SCR Manual. New York: General Electric Company, 1972.

[7] "Hybrid Switch," U.K. International Application PCT/GB96/02700.

[8] S. Greitzke and M. Lindmayer, "Commutation and erosion in hybrid switch systems.," IEEE Trans. Compon., Hybrids Manufact. Technol., vol. CHMT-8, no. 1, pp. 34-39, 1985.

[9] S. Greitzke and M. Lindmayer, "Phenomena of microerosion in contacts shunted by semiconductor devices," Elektrotech. Informationstech., vol. 107, no. 2, pp. 57-62, 1990.

[10] P. J. Theisen, S. Krstic, and C. G. Chen, " $240 \mathrm{~V}$ dc hybrid switch.," in Proc. IEEE Holm Conf. Elect. Contacts, 1985, pp. 75-79.

[11] F. Tofigh, "208 V AC, $20 \mathrm{kHz}$ hybrid remote power controller.," in Proc. 22nd Intersoc. Energy Conv. Eng. Conf., 1987, pp. 10-14.

[12] S. Krstic and P. J. Theisen, "Push Button Hybrid Switch.," in Proc. IEEE Holm Conf. Electrical Contacts, 1985, pp. 201-205.

[13] F. Lauerer, "Elektonisch-mechanischer schalter," DE3341947 A1, 1983.

[14] I. S. Kim, "Arc-Free Contactor," U.K. Patent Application GB 2191 341 A, 1987.

[15] [Online]. Available: www.taicaan.com

[16] J. W. McBride, G. J. Witter, and Z. K. Chen, "Volumetric erosion measurements of contacts tested in dc automotive relays," in Proc. IEEE Holm. Conf. Elect. Contacts, 2001, pp. 73-81.

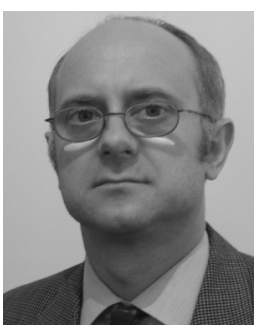

Jonathan Swingler received the M.S. degree in physics and chemistry from the University of Keele, U.K., in 1990 and the Ph.D. degree in the degradation of electrical contacts under low frequency fretting conditions from Loughborough University, Loughborough, U.K., in 1994.

Since then, he has been pursuing research in the area of electrical contact science and technology at the University of Southampton, Southampton, U.K., and is currently a Lecturer in the Electro-Mechanical Research Group. He lectures undergraduate Electrical Systems, Electromechanical Machine, and Automotive Electronics.

Dr. Swingler is a Chartered Scientist, Chartered Physicist, and a Member of the Institute of Physics.

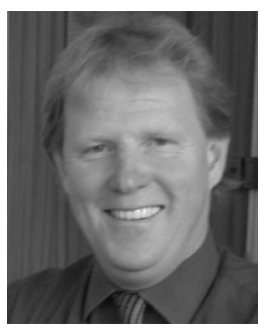

John W. McBride received the M.S. degree in aeronautical engineering from the University of Southampton, Southampton, U.K., in 1978 and the $\mathrm{Ph} . \mathrm{D}$. degree in electrical contact phenomena from Plymouth University, Plymouth, U.K., in 1986.

$\mathrm{He}$ is a Professor of electro-mechanical engineering in the School of Engineering Science, University of Southampton, where he is Chair of the Electro-Mechanical Research Group, and Director of Research. His main research interests include electrical contacts, metrology, and instrumentation.

Dr. McBride is an Associate Editor of the IEEE TRANSACTIONS ON COMPONENTS AND PACKAgING TeChNOLOGIES and a member of the Organizing committee for the IEEE Holm Conference on Electrical Contacts. 